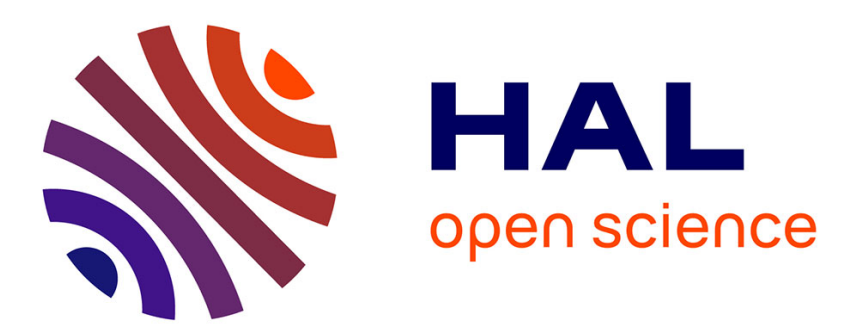

\title{
Mapping and localization using GPS, lane markings and proprioceptive sensors
}

Zui Tao, Philippe Bonnifait, Vincent Fremont, Javier Ibañez-Guzman

\section{To cite this version:}

Zui Tao, Philippe Bonnifait, Vincent Fremont, Javier Ibañez-Guzman. Mapping and localization using GPS, lane markings and proprioceptive sensors. IEEE/RSJ International Conference on Intelligent Robots and Systems (IROS 2013), Nov 2013, Tokyo, Japan. pp.406-412. hal-00880566

\section{HAL Id: hal-00880566 https://hal.science/hal-00880566}

Submitted on 6 Nov 2013

HAL is a multi-disciplinary open access archive for the deposit and dissemination of scientific research documents, whether they are published or not. The documents may come from teaching and research institutions in France or abroad, or from public or private research centers.
L'archive ouverte pluridisciplinaire HAL, est destinée au dépôt et à la diffusion de documents scientifiques de niveau recherche, publiés ou non, émanant des établissements d'enseignement et de recherche français ou étrangers, des laboratoires publics ou privés. 


\title{
Mapping and localization using GPS, lane markings and proprioceptive sensors
}

\author{
Z. Tao ${ }^{1,2}$, Ph. Bonnifait ${ }^{1,2}$, V. Frémont ${ }^{1,2}$, J. Ibañez-Guzman ${ }^{3}$
}

\begin{abstract}
Estimating the pose in real-time is a primary function for intelligent vehicle navigation. Whilst different solutions exist, most of them rely on the use of high-end sensors. This paper proposes a solution that exploits an automotive type L1GPS receiver, features extracted by low-cost perception sensors and vehicle proprioceptive information. A key idea is to use the lane detection function of a video camera to retrieve accurate lateral and orientation information with respect to road lane markings. To this end, lane markings are mobile-mapped by the vehicle itself during a first stage by using an accurate localizer. Then, the resulting map allows for the exploitation of camera-detected features for autonomous real-time localization. The results are then combined with GPS estimates and deadreckoning sensors in order to provide localization information with high availability. As L1-GPS errors can be large and are time correlated, we study in the paper several GPS error models that are experimentally tested with shaping filters. The approach demonstrates that the use of low-cost sensors with adequate data-fusion algorithms should lead to computercontrolled guidance functions in complex road networks.
\end{abstract}

\section{INTRODUCTION}

Localization is an important issue in current intelligent vehicles research activities. An accurate and reliable localization is the basis of vehicle navigation. A vast number of localization methods can be found in the literature. A comprehensive overview is given in [1].

If no a priori map data is used in the localization process, methods can be divided into two categories: GPS/INS integration method [2] and Simultaneous Localization And Mapping (SLAM) methods. The benefits of integrating GPS with INS are that INS may be calibrated by GPS signals and can provide position and orientation updates at a quicker rate than GPS. GPS may lose its signal due to outages. INS can continue to compute the position and orientation during the period of lost of the GPS signal. Performance of GPS/INS integration method mainly depends on the quality of INS, but a high-performance INS is usually expensive. Deadreckoning (DR) sensors already installed on automobiles can be used instead. SLAM enables the vehicle to build an online map and update the map over time. SLAM has been used a lot with laser scanners. Its feasibility has been proved in outdoor experiments [3], [4].

Nowadays, with the development of refined digital maps, vision-based and map-aided vehicle localization has been a research trend, in combination with GPS and proprioceptive sensors. Many methods have been proposed in literature. [5] develops an algorithm which directly transforms map feature data into an image of map features, the incorporation of

The authors are with ${ }^{1}$ Université de Technologie de Compiègne (UTC), ${ }^{2}$ CNRS Heudiasyc UMR 7253, ${ }^{3}$ Renault S.A.S, France.

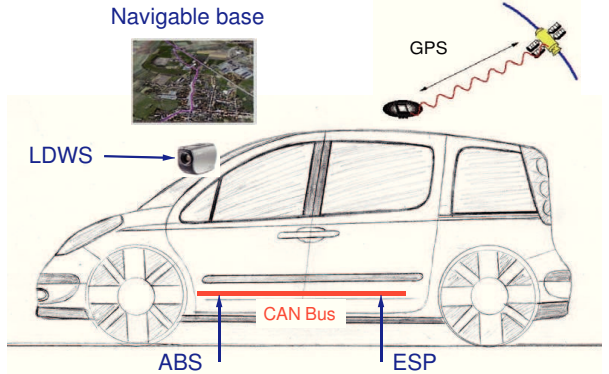

Fig. 1. Automotive systems used for mapping and localization

the map information is moved from feature level to signal level. [6] integrated OpenStreetMap data into the robot tasks of localization, and in [7] a multi-camera system has been developed for outdoor localization.

In this paper, we use automotive sensors that have CANbus interfaces. In Europe, every new vehicle must be equipped with an Electronic Stability Program (ESP) since January 2012, and Anti-lock Braking System (ABS) has been part of standard equipments in earlier times. These two systems contain a wealth of proprioceptive sensors which are capable of measuring vehicle rotation, individual wheel speeds and lateral acceleration, etc.. These sensors can be used as DR sensors. Modern automobiles (Fig. 1) can be also often equipped with a Lane Departing Warning System (LDWS) and turn-by-turn GPS navigation. LDWS provides on the CAN bus lane type, quality of the detection, lane position, lane marking's curvature, curvature derivative and host heading relative to lane marking. The GPS used by the turn-by-turn navigation system is reused by our localization system. Therefore, the localization system studied in this paper reuse sensors already installed for other automotive functions.

The fusion of all these existing sensors gives the possibility to improve the localization and navigation performance. Integration of a low cost L1-GPS receiver (mono-frequency) and DR sensors is quite easy to achieve but provides insufficiently accurate results. In order to integrate exteroceptive perception of the environment in the vicinity, we use a video camera that detects lane markings since this kind of feature is well adapted to road conditions. Moreover, we are interested in applications where the vehicle can learn a precise map (currently available commercial maps present serious lacks of accuracy, contents and completeness to be applicable in high-demanding applications) when driven by a human operator in a first stage. Then, the vehicle uses this map for autonomous navigation, so SLAM is not necessary. 
Moreover, during the learning stage the vehicle can exploit an accurate localization system in order to get an accurate map. This accurate localization system is not needed any more when the vehicle navigates autonomously since it is able to infer an accurate positioning using its map and an embedded exteroceptive sensor like a camera. So, this paper proposes to use road lane marking information and an a priori digital map of the lane markings which is made by mobile mapping [8]. An observation model of camera measurement is proposed in section II-D. The lane detection result provides accurate localization information which is both redundant and complementary with GPS information. Unfortunately, due to the fact that lane markings are not always available on roads, GPS information has to be used as much as possible even if it can be affected by large errors which can affect deeply the result of the data fusion process. So, a crucial issue is to model and estimate GPS errors. We study in this paper several GPS error models and we prove that each of them is observable. Shaping filters are then used in the localizer.

The paper is organized as follows. Section II introduces the system modeling with the model of camera measurement. Section III describes the mobile mapping process for building a digital map of the lane markings. Section IV gives a description of three GPS error models. After having described the localizer that performs the data fusion, results of real outdoor experiments are presented in section VI. Conclusions on system performance and GPS error modeling are given in section VII.

\section{SySTEM MODELING}

\section{A. Navigation frame}

As the system exploits global GPS information in geographic coordinates, a local navigation frame $R_{O}$ tangent to the Earth is useful to manipulate classical Cartesian equations.

$R_{O}$ is defined to have its $x$ axis pointing to the East, $y$ to the North and $z$ oriented upwards with respect to the WGS84 ellipsoid.

Its origin can be any GPS position fix close to the navigation area. Moreover, as this point can be chosen arbitrarily close to the navigation area, one can suppose that the trajectory is planar in 2D.

Any mapping or localization problem will be expressed, in the following, in this local coordinate system.

\section{B. Frames attached to the mobile}

Two more frames have to be defined (see Fig. 2). $R_{M}$ denotes the mobile vehicle reference frame $\left(x_{M}\right.$ is the longitudinal axis pointing forward and $y_{M}$ is such that $z_{M}$ is upwards). Point $C$, the origin of the camera frame $R_{C}$, is located at the front of the vehicle since camera systems do often auto-calibration. In order to stay consistent with vision system conventions, $y_{C}$ is right-hand oriented. Even if the camera is located behind the windscreen with a position offset $\left(C_{x}, C_{y}\right)$, every detected lane marking is expressed in $R_{C}$.
$P_{x}$ is a translation from point $M$ up to the bumper. Let denote ${ }^{M} T_{C}$ the homogeneous transformation matrix mapping $R_{C}$ in $R_{M}$ :

$$
{ }^{M} T_{C}=\left[\begin{array}{ccc}
1 & 0 & P_{x} \\
0 & -1 & 0 \\
0 & 0 & 1
\end{array}\right]
$$

The transformation ${ }^{O} T_{M}$ maps $R_{M}$ in $R_{O}$ :

$$
{ }^{O} T_{M}=\left[\begin{array}{ccc}
\cos \theta & -\sin \theta & x \\
\sin \theta & \cos \theta & y \\
0 & 0 & 1
\end{array}\right]
$$

Let define $q=(x, y, \theta)^{T}$ the vehicle's pose which includes position $(x, y)$ and heading angle $\theta$.

\section{Kinematic model}

Under the assumption that the rear wheels do not slip, the speed vector is collinear to $x_{M}$, which conducts to the classical unicycle model:

$$
\left\{\begin{array}{l}
\dot{x}=v \cdot \cos \theta \\
\dot{y}=v \cdot \sin \theta \\
\dot{\theta}=\omega
\end{array}\right.
$$

where the inputs are the linear velocity $v$ and the angular velocity $\omega$. They are measured by the wheel speed sensors (ABS system) and the yaw rate gyro (ESP system). They are provided to several sub-systems of the vehicle through the CAN bus.

As we use a low cost yaw rate gyro, its drift rate $\varepsilon_{\omega}$ is modeled by a random constant [9]. The dynamic model becomes:

$$
\left\{\begin{array}{l}
\dot{x}=v \cdot \cos \theta \\
\dot{y}=v \cdot \sin \theta \\
\dot{\theta}=\omega-\varepsilon_{\omega} \\
\dot{\varepsilon_{\omega}}=0
\end{array}\right.
$$

Even if the drift rate $\varepsilon_{\omega}$ is affecting an input of the system, it is easy to prove its observability with a lateral distance measurement.

\section{Camera measurements}

We consider a camera system which provides the following lane marking parameters of a Taylor's expansion of a clothoid in the camera frame [10]:

$$
y=C_{3} \cdot x^{3}+C_{2} \cdot x^{2}+C_{1} \cdot x+C_{0}
$$

Where $C_{0}, C_{1}, C_{2}$ and $C_{3}$ are respectively the lateral distance, the slope, the curvature and the curvature derivative of the detected lane marking.

The parameters that are used for vehicle localization are the lateral distance $C_{0}$ and the heading $C_{1}$ (see Fig. 2). Observations provided by the camera system are denoted $Y_{\text {cam }}=\left[C_{0}, C_{1}\right]^{T}$.

Let us establish observation models between $Y_{\text {cam }}$ and the pose vector $q$.

In Fig. 2, $[\mathrm{AB}]$ is the detected lane marking segment which has been extracted from the digital map by a map matching process. In $R_{O}$, the equation of segment [AB] is: 


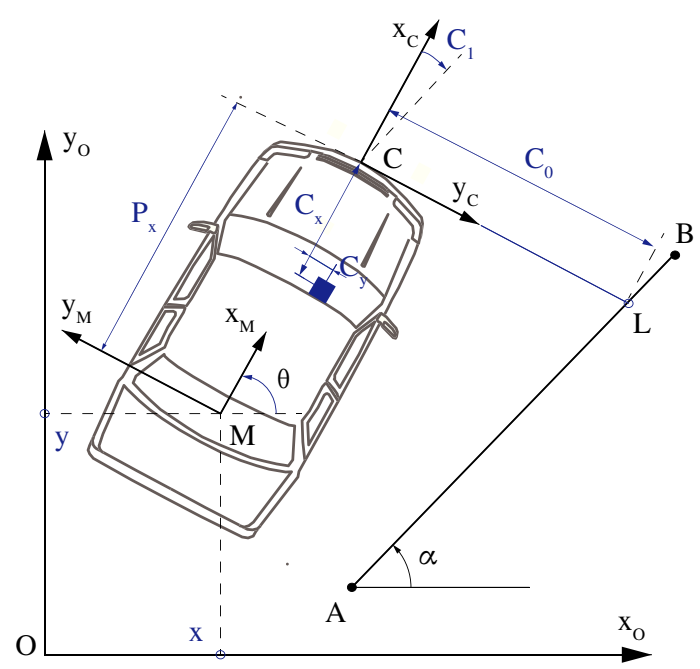

Fig. 2. Frames. $[\mathrm{AB}]$ is a detected lane marking segment.

$$
y=a \cdot x+b
$$

where $a$ and $b$ are the line coefficients. Let $\alpha$ be the slope angle of segment $[\mathrm{AB}], \alpha \in\left[-\frac{\pi}{2}, \frac{\pi}{2}\right]$.

$$
C_{1}= \begin{cases}\alpha-\theta, & |\alpha-\theta| \leq \frac{\pi}{2} \\ \alpha-\theta+\pi \cdot \operatorname{sign}(\theta), & |\alpha-\theta|>\frac{\pi}{2}\end{cases}
$$

In $R_{C}$, the homogeneous coordinates of point $L$ (see Fig. 2) are ${ }^{C} L=\left[0, C_{0}, 1\right]^{T}$. So, ${ }^{O} L={ }^{O} T_{M}{ }^{M} T_{C} \cdot{ }^{C} L$ are the homogeneous coordinates of point $L$ in frame $R_{O}$ :

$$
{ }^{O} L=\left[\begin{array}{c}
P_{x} \cdot \cos \theta+C_{0} \cdot \sin \theta+x \\
P_{x} \cdot \sin \theta-C_{0} \cdot \cos \theta+y \\
1
\end{array}\right]
$$

Because point $L$ is on the segment $[\mathrm{AB}],{ }^{O} L$ verifies the equation of the line $y=a \cdot x+b$ which gives:

$$
C_{0}=\frac{P_{x} \cdot \sin \theta-a \cdot P_{x} \cdot \cos \theta-a \cdot x+y-b}{a \cdot \sin \theta+\cos \theta}
$$

Please notice that the singularity due to a division by zero means that the detected line is perpendicular to the vehicle which can never occur in practice.

\section{MOBILE MAPPING}

The positioning system needs an accurate digital map of the road network. For real-time efficiency, the map consists of line segments which represent the lane markings on the road. By definition, mobile mapping is the process of collecting geospatial data from a mobile vehicle, typically fitted with a range of photographic, radar, laser or any number of remote sensing systems. Such systems are composed of a set of time synchronized navigation sensors and imaging sensors mounted on a mobile platform. Mobile mapping is often assisted by a human operator.

The mobile mapping system used in this approach only needs to equip the vehicle with a RTK-GPS receiver aided by an Inertial Measurement Unit (IMU) in order to get

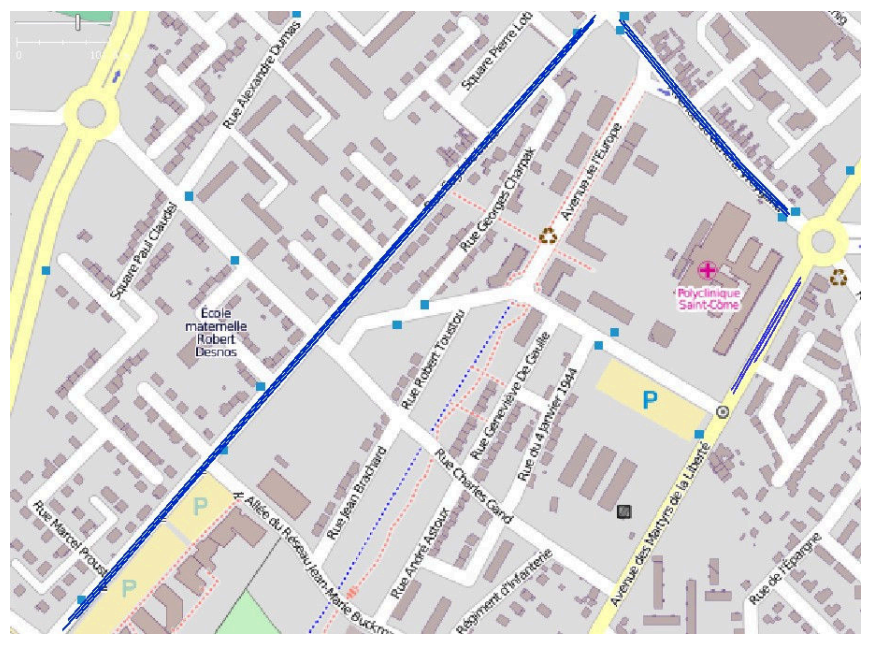

Fig. 3. Map of a test site superimposed on an Open Street Maps image

an accurate pose estimation of the body frame. The vision system (the same one as the system used by the localization system) detects lane markings and provides parameters of the clothoids that are recorded during the trial.

As mobile mapping is done in post-processing, it is quite easy to synchronize the positioning data with the measurements of the camera by interpolating the trajectory after having compensated the clock drift of the acquisition system with respect to the GPS. This allows georeferencing every detected lane marking point $L$ in $R_{O}$.

Let denote $\left[x_{L}, y_{L}\right]^{T}$ the coordinates of point $L$ in $R_{O}$. It can be mapped using (Eq.7) which gives:

$$
\left[\begin{array}{l}
x_{L} \\
y_{L}
\end{array}\right]=\left[\begin{array}{l}
P_{x} \cdot \cos \theta+C_{0} \cdot \sin \theta+x \\
P_{x} \cdot \sin \theta-C_{0} \cdot \cos \theta+y
\end{array}\right]
$$

It can provide a huge amount of data, which will not be efficient for real-time navigation, to keep every mapped lane marking point in the map. Therefore, the obtained lane marking points are vectorized and filtered to build a precise vector map by using the Douglas-Peucker's algorithm [11] and a least squares algorithm. Douglas-Peucker's algorithm is a well known algorithm for reducing the number of points in a curve that is approximated by a series of points. It's used here to divide the lane marking points into parts with different slopes. The origin set of points $p$ is simplified to $p s$ (vertexes of simplified line) with a certain tolerance (maximal euclidean distance allowed between the simplified line and an origin point).

As this segmentation algorithm is noise sensitive, a least squares algorithm is then performed on the lane marking points between the two endpoints of every simplified line in order to increase the accuracy of the map. Let $\left[p s_{1} p s_{2}\right]$ be one of these simplified lines. If $p l$ denotes every lane marking points between points $p s_{1}$ and $p s_{2}$, by using a least squares fitting on $p l$, one gets a new estimation of the line. The intersection of the new estimated lines provides new segment $\left[p s_{1}^{\prime} p s_{2}^{\prime}\right]$ which is finally stored in the digital map (blue lines in Fig. 3). 

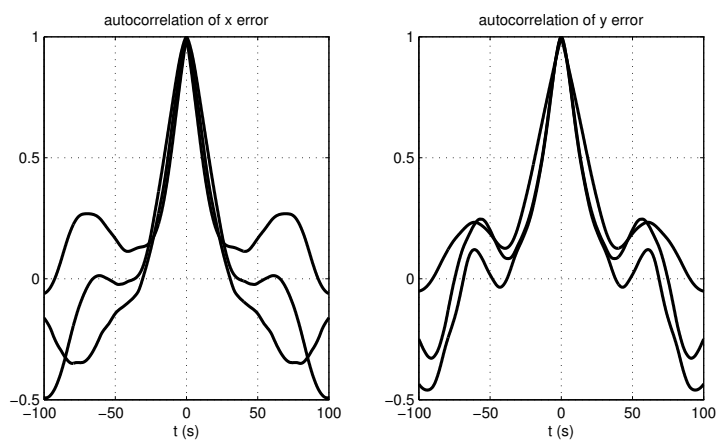

Fig. 4. Autocorrelation of different sequences

\section{GPS ERRORS}

As well known, loosely coupling L1-GPS ${ }^{1}$ with other sensors is a challenging task since GPS positioning errors are not white, can be affected by strong biases (due to atmosphere propagation delays) and multi-path, particularly in urban areas. Fig. 12 (the red line displays L1-GPS position errors) illustrates these issues: wrong biases can affect estimated fixes for significant duration and positioning errors often reach few meters.

Anyway, GPS positioning is useful for system initialization and when there is no lane marking to correct the drift of DR. Therefore, we study here three different models of GPS errors and compare their performance. Recent work on GPS bias correction can be found in [12].

\section{A. GPS error models}

Let suppose that L1-GPS position errors are stationary. Autocorrelations of 3 different sequences of a low cost L1GPS receiver (1000 samples each at $5 \mathrm{~Hz}$ ) are shown in Fig. 4. Clearly, errors are colored since there is no Delta-Dirac at zero. Moreover, for short correlation times (smaller than 30 seconds), the different curves superimpose quite well which indicates a quite repeatable behavior which can be modeled by a shaping filter. Here, we suggest to model the correlation by a first order regressive system, the autocorrelation of which is a decreasing exponential. The time constant is simply given by the intersection of the initial tangent with the final asymptote which gives here $25 \mathrm{~s}$ in $x$ and in $y$.

Let $\left[\begin{array}{cc}\varepsilon_{x}, & \varepsilon_{y}\end{array}\right]^{T}$ denote the vector of GPS errors. A classical way to handle this kind of modeling is to augment the state vector with a shaping filter [9]:

$$
X=\left[\begin{array}{llllll}
x, & y, & \theta, & \varepsilon_{\omega} & \varepsilon_{x}, & \varepsilon_{y}
\end{array}\right]^{T}
$$

And to use a first order auto-regressive process:

$$
\text { Model } 1\left\{\begin{array}{l}
\dot{\varepsilon}_{1, x}=-\varepsilon_{1, x} / \tau_{x}+v_{\varepsilon_{x}} \\
\dot{\varepsilon}_{1, y}=-\varepsilon_{1, y} / \tau_{y}+v_{\varepsilon_{y}}
\end{array}\right.
$$

Where $v_{\varepsilon_{x}}$ and $v_{\varepsilon_{y}}$ are the driving white noises of the shaping filter. $\tau_{x}$ and $\tau_{y}$ are the time constants. Time constants of the shaping filter give an idea of the time required for the system to be in steady state.

GPS error can be also modeled by a random constant, where $\alpha_{\varepsilon_{i}}$ is a zero mean white noise:

\footnotetext{
${ }^{1}$ L1-GPS means mono-frequency receiver with C/A pseudo-ranges
}

$$
\text { Model } 2\left\{\begin{array}{l}
\dot{\varepsilon}_{2, x}=0+\alpha_{\varepsilon_{x}} \\
\dot{\varepsilon}_{2, y}=0+\alpha_{\varepsilon_{y}}
\end{array}\right.
$$

Finally, a more refined model of the error is to combine both of them:

$$
\operatorname{Model} 3\left\{\begin{array}{l}
\varepsilon_{x}=\varepsilon_{1, x}+\varepsilon_{2, x}+\alpha_{\varepsilon_{x}} \\
\varepsilon_{y}=\varepsilon_{1, y}+\varepsilon_{2, y}+\alpha_{\varepsilon_{y}}
\end{array}\right.
$$

In this latter case, the length of vector $\mathrm{X}$ is augmented up to 6 components.

\section{B. Observability of GPS errors}

Since GPS component errors are additive, their observability is not obvious. We prove here that this is the case.

A system variable $x$ is said to be observable, if and only if there is an algebraic equation linking $x$, the outputs, the inputs and a finite number of the derivatives of inputs and outputs. This algebraic definition of observability was in [13] to prove that angular measurements provided by a camera are enough to perform localization in challenging conditions. For non-linear systems, this definition of observability is more powerful than geometrical concepts based on Lie derivatives since it provides necessary and sufficient conditions [14]. In order to prove the observability of GPS measurement errors, let us simplify the state-space model by :

- Supposing that point $C$ and the GPS antenna are coinciding with point $M$;

- Ignoring the gyro drift rate.

Moreover, one can do an interesting frame-to-frame transformation by choosing the origin point $O$ of $R_{o}$ on the lane marking and $x$ axis of $R_{o}$ parallel to the lane marking.

This simplification yields $P_{x}=0, a=0, b=0$ and $\dot{\theta}=\omega$, and the state vector becomes:

$$
X=\left[\begin{array}{lll}
y, & \theta, & \varepsilon_{y}
\end{array}\right]^{T}
$$

Consequently, (Eq. 8) and GPS observation are simplified to:

$$
C_{0}=y / \cos \theta
$$

and

$$
y_{G P S}=y+\varepsilon_{y}
$$

By taking the derivative of (Eq. 13), we have:

$$
\dot{y}=\dot{C}_{0} \cdot \cos \theta-\omega \cdot C_{0} \cdot \sin \theta
$$

By plugging $\dot{y}=v \cdot \sin \theta$ into (Eq. 15), we can obtain:

$$
\theta=\arctan \left(\frac{\dot{C}_{0}}{(v+\omega) \cdot C_{0}}\right)
$$

So $\theta$ is observable, thus $y$ is observable by (Eq. 13) and $\varepsilon_{y}$ is observable by (Eq. 14).

If we use now the double-error model (Eq. 12), we get $\dot{\varepsilon}_{y}=\dot{\varepsilon}_{1, y}+\dot{\varepsilon}_{2, y}$. By plugging (Eq. 10) and (Eq. 11) into this equation, we get:

$$
\dot{\varepsilon}_{y}=-\varepsilon_{1, y} / \tau_{y}+0
$$


So $\varepsilon_{1, y}$ is observable and thus $\varepsilon_{2, y}=\varepsilon_{y}-\varepsilon_{1, y}$ is observable.

As a consequence, the two GPS errors are observable and so reconstructable by any convergent state observer, like a Kalman filter for instance.

In a similar way, one can prove the observability of GPS errors on $x$ when the lane marking is North oriented. This shows that, even if the lane marking measurements provide essentially a lateral correction, the GPS errors are observable as soon as the trajectory presents changes of direction.

\section{LOCALIZATION SOLVER IMPLEMENTATION}

For an accurate data fusion, the lever arm has to be taken into account. The position of the L1-GPS antenna in $R_{M}$ is denoted by ${ }^{M} G=\left[t_{x}, t_{y}\right]^{T}$. An efficient way to compensate for the latency GPS fixes (denoted $\Delta t$ ) is to extrapolate the fix at the right time using the speed. The GPS observation model becomes:

$$
\left\{\begin{array}{l}
x_{G P S}=\cos \theta \cdot\left(t_{x}-\Delta t \cdot v\right)-\sin \theta \cdot t_{y}+x+\varepsilon_{x} \\
y_{G P S}=\sin \theta \cdot\left(t_{x}-\Delta t \cdot v\right)+\cos \theta \cdot t_{y}+y+\varepsilon_{y}
\end{array}\right.
$$

The evolution and camera models have been described in section II.

Many solvers can be used to do the estimation problem (e.g. Extended Kalman filter EKF, Particle filter and Unscented Kalman filter). In this paper, a full-state EKF with measured inputs $v$ and $\omega$ is adopted (the covariance matrix of the input noise is propagated into the computation). As it is triggered by the CAN frequency $(100 \mathrm{~Hz})$, the EKF is also "time continuous" with discrete exteroceptive measurements. Camera measurements are used in estimation stages after having been map-matched. An innovation gate is also applied to reject outliers due to wrong matching or bad detections. The same rejection strategy is adopted for the GPS data. We also use GPS only when the speed is non zero, since it is well known that satellite multi-path is badly rejected by GPS correlators at low speed.

\section{RESULTS}

\section{A. Experimental set-up}

Outdoor experiments have been carried out in the city of Compiègne (France). A digital map of the lane markings of the experimental road (Fig. 3) has been made by using the method described in section III in May 2012. We report here experiments realized in February 2013 (9 months later).

The approach detailed in this paper has been tested with the experimental vehicle showed in Fig. 5.

The vehicle was equipped with a NovAtel RTK-GPS receiver coupled with a SPAN-CPT IMU running at $10 \mathrm{~Hz}$. The system received RTCM 3.0 corrections through a $3 \mathrm{G}$ connection from a GPS base station Septentrio PolaRx2e@ equipped with a Zephyr Geodetic antenna. This high accuracy system (few tens of centimeter) was used during the mobile mapping process. It also provided ground truth data for the localization method. The station was located at the research center in Compiègne. Its position was the origin

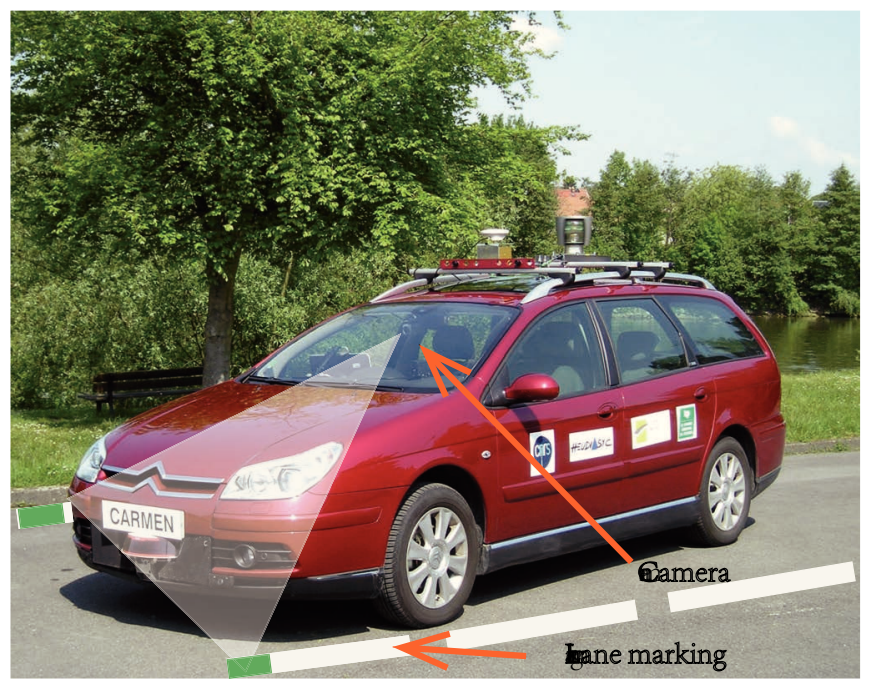

Fig. 5. Experimental vehicle. The camera is located behind the windscreen.

$O$ of the local coordinate system (latitude $49.4^{\circ}$, longitude $2.796^{\circ}$, ellipsoidal height $83 \mathrm{~m}$ ). A CAN-bus gateway was used to access to wheel speed sensors (WSS) and to a yaw rate gyro. A Mobileye camera was used to detect the lane markings, on the basis of lane detection function of Mobileye's Lane Keeping and Guidance Assistance System (LKA). The output is described in Mobileye's LKA common CAN protocol. The low-cost GPS was a U-blox 4T with a patch antenna.

Fig. 6 shows the ground truth of the test path and some images taken during the mobile mapping. During the localization test, it should be noticed that the weather was rainy. The vehicle was normally driven. Three stops and a backward maneuvered occurred.

A point-to-curve map-matching process [15] with distance, direction and heading was used to identify the correct lane marking segments. This was applied on right and left detections. Moreover, a lane change has been done because of the overtaking of a bus. This was particularly useful to test the map-matching performance.

\section{B. Localization results}

The different filters have been tested by using data replay. The initial state given to the filter was the first L1-GPS fix and the components of the GPS errors were initialized to zero in order to respect real-time conditions.

Fig. 8 shows localization errors on $x$ and $y$ by only fusing DR sensors and camera. The red line is the $\pm 3 \sigma$ confidence interval estimated by the EKF. The blue and green points separately indicate lane detection on left and right side. On one hand, the camera provides efficient information to correct the DR drift without GPS. On the other hand, DR predictions are useful to estimate the vehicle pose when there is no lane marking (typically near junctions).

Fig. 9, 10 and 11 show localization errors on $x$ and $y$ when fusing DR sensors, camera and L1-GPS with the different models (first order auto-regressive process, random constant 

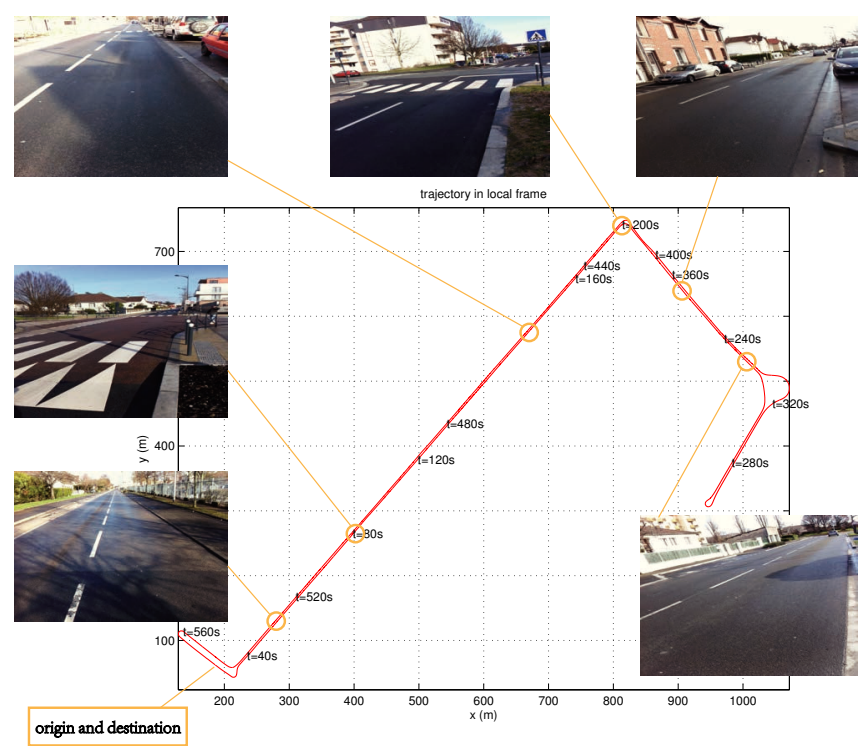

Fig. 6. Test trajectory. A speed bumper is visible at time $\mathrm{t}=80 \mathrm{~s}$.

\begin{tabular}{|l|c|c|c|}
\hline \multirow{2}{*}{} & \multicolumn{3}{|c|}{ Horizontal positioning errors (m) } \\
\cline { 2 - 4 } & Model 1 & Model 2 & Model 3 \\
\hline \hline mean & 1.82 & 1.57 & 1.21 \\
\hline std. dev. & 0.81 & 1.01 & 0.75 \\
\hline max & 4.62 & 3.44 & 3.04 \\
\hline median & 1.79 & 1.35 & 1.03 \\
\hline 95th percentile & 3.69 & 3.38 & 2.59 \\
\hline
\end{tabular}

TABLE I

STATISTICS FOR THE FILTER WITH THE THREE GPS ERROR MODELS

and combination of both). In each figure, errors are plotted with $\pm 3 \sigma$ bounds to show the well tuning of the filters.

Performance metrics of the different error models are given in Table I. Globally speaking, Model 2 is better than Model 1 and Model 3 gives the best performance. The accuracy gain obtained by an adequate GPS errors model is very significant compared to Fig. 12.

In Fig. 12, the red line displays the real L1-GPS position errors with respect to the SPAN-CPT. The blue, green and black lines correspond respectively to the estimated GPS position errors of Model 1,2 and 3. They confirm the results of Table I.

Let us now study more in detail the lateral and longitudinal errors when using Model 3 (see Table II). Logically, the lateral accuracy is much better than the longitudinal one (e.g. $95 \%$ of the lateral error is less than $30 \mathrm{~cm}$ ). The maximum error occurs at the strong U-turn which was performed with a very small radius of curvature with no lane marking detection. Moreover, one can notice that longitudinal error is also controlled by taking profit of the heading variation of the trajectory. This is verified by the fact that the trajectory was making a loop (the vehicle came back at its starting) and the error between the starting and the end points is $0.31 \mathrm{~m}$. We believe that this accuracy is sufficient for autonomous navigation on normal roads.

Fig. 7 gives an illustration of the system at a junction. It can be seen that the DR drift is quickly corrected as soon as map-matched camera measurements are available. Moreover,

\begin{tabular}{|l|c|c|}
\hline & lateral error $(\mathrm{m})$ & longitudinal error $(\mathrm{m})$ \\
\hline \hline mean & 0.11 & 1.08 \\
\hline std. dev. & 0.12 & 0.69 \\
\hline max & 1.03 & 2.78 \\
\hline median & 0.07 & 0.91 \\
\hline 95th percentile & 0.30 & 2.50 \\
\hline
\end{tabular}

TABLE II

ERROR STATISTICS WITH MODEL 3

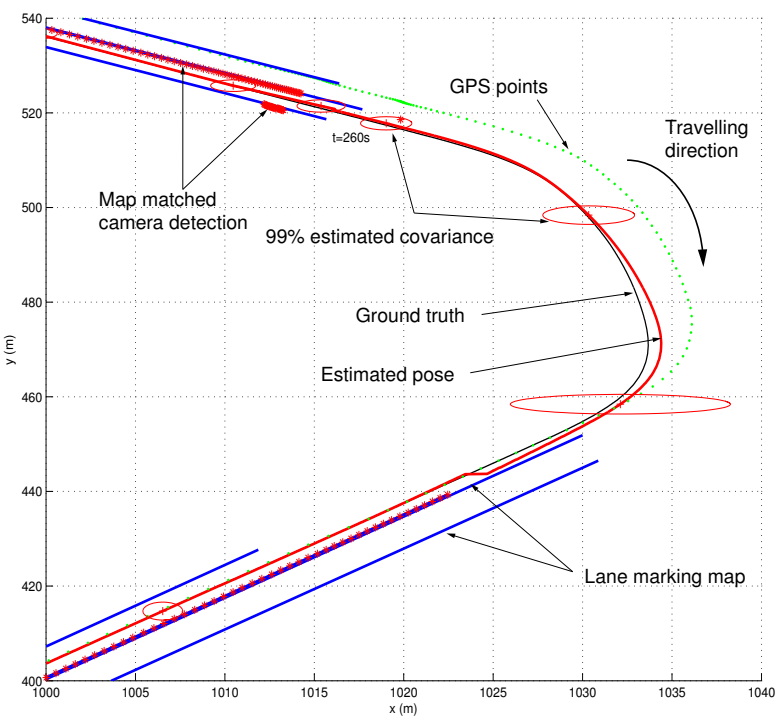

Fig. 7. Bird view zoom around $t=260 \mathrm{~s}$

the position estimate stays in the correct lane and the large GPS errors are well managed by the filter.

\section{CONCLUSION}

This paper has presented a new vehicle localization method which fuses L1-GPS, CAN-bus sensors, video camera and digital map data. Since there is no ready-made digital map which contains the lane marking information, a digital map of the lane markings has been made by mobile mapping. Moreover, the method uses natural features and so does not need any change of the road environment. Precise observation models of the vision system have been proposed in order to correct efficiently the DR drift but also GPS errors. Moreover, three GPS error models have been compared. The GPS shaping filter combining random constant and first order auto-regressive models gives good results with a limited computational complexity. Thanks to this modeling, the filter is able to fuse continuously GPS fixes even if they can be affected by large errors. Finally, real results have shown that the proposed method can improve the lateral localization up to a centimeter-level accuracy with low cost sensors already installed on modern vehicles.

\section{ACKNOWLEDGMENTS}

The authors would like to thank N. Chaari, A. Miranda Neto and G. Dherbomez for their help in the experiments.

\section{REFERENCES}

[1] I. Skog and P.Handel, "In-car positioning and navigation technologiesa survey," IEEE Transactions on Intelligent Transportation Systems, vol. 10, no. 1, pp. 4-21, 2009. 


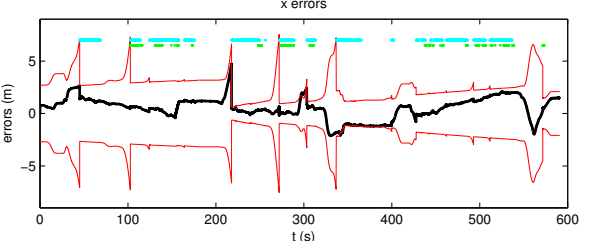

y errors

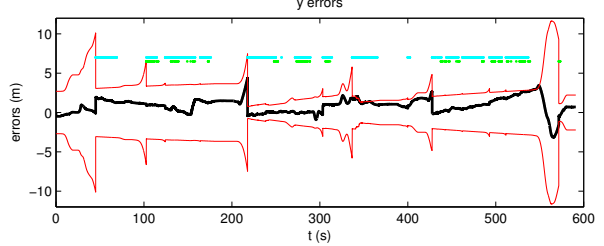

Fig. 8. Position errors without GPS

[2] K.A.Redmill, T.Kitajima, and U.Ozgumer., "Dgps/ins integrated positioning for control of automated vehicle," Proc IEEE Int. Transp. Systems, pp. 172-178, 2001.

[3] M. Dissanayake, P. Newman, S. Clark, H. Durrant-Whyte, and M. Csorba, "A solution to the simultaneous localization and map building (slam) problem," IEEE Transactions on Robotics and Automation, vol. 17, no. 3, pp. 229-241, 2001.

[4] J. Guivant, F. Masson, and E. Nebot, "Simultaneous localization and map building using natural features and absolute information," Robotics and Autonomous Systems, vol. 40, no. 2-3, pp. 79-90, Aug. 2002.

[5] Mattern, N.Schubert, R.Wanielik, and G.Fac, "High-accurate vehicle localization using digital maps and coherency images," Int. Vehicles Symp., 2010

[6] M. Hentschel and B. Wagner, "Autonomous robot navigation based on openstreetmap geodata," Madeira Island, Portugal, 2010, pp. 16451650 .

[7] I. Miller, M. Campbell, and D.Huttenlocher, "Map-aided localization in sparse global positioning system environments using vision and particle filtering," Journal of Field Robotics, vol. 28, no. 5, pp. 619643, 2011.

[8] H. Gontran, J. Skaloud, and P. Gillieron, "A mobile mapping system for road data capture via a single camera," Proceedings of the Optical $3 D$ Conference, 2003.

[9] Y. Bar-shalom, X.-R. Li, and T. Kirubarajan, Estimation with Applications to Tracking and Navigation. New York, NY, USA: John Wiley \& Sons, Inc., 2002.

[10] K. Kluge, "Extracting road curvature and orientation from image edge points without perceptual grouping into features," Int. Vehicles Symp., pp. 109-114, 1994.

[11] D. Douglas and T. Peucker, "Algorithms for the reduction of the number of points required to represent a digitized line or its caricature," Cartographica: The International Journal for Geographic Information and Geovisualization, vol. 10, no. 2, pp. 112-122, 1973.

[12] K. Jo, K. Chu, and M. Sunwoo, "Gps-bias correction for precise localization of autonomous vehicles," Int. Vehicles Symp., pp. 636641, June 2013.

[13] H. Sert, W. Perruquetti, A. Kokosy, X. Jin, and J. Palos, "Localizability of unicycle mobiles robots: An algebraic point of view," IEEE Conf. on Int. Robots and Systems, pp. 223-228, 2012.

[14] S. Wijesoma, K. W. Lee, and J. I. Guzman, "On the observability of path constrained vehicle localisation," IEEE Conf. on Int. Transp. Systems, pp. 1513-1518, 2006.

[15] M. El Badaoui El Najjar and P. Bonnifait, "A road-matching method for precise vehicle localization using kalman filtering and belief theory," Journal of Autonomous Robots, vol. Volume 19, no. Issue 2, pp. 173-191, September 2005.

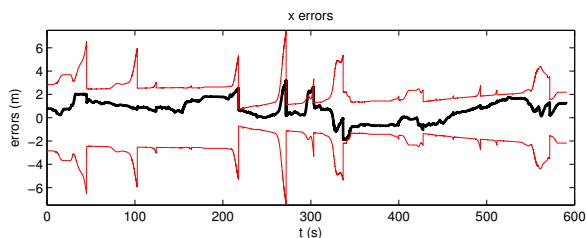

y errors

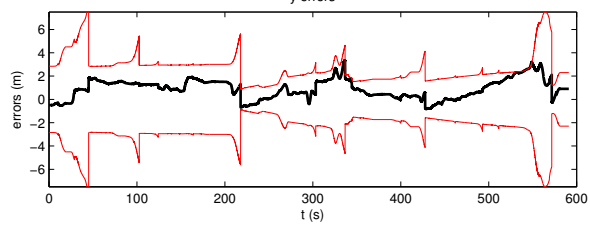

Fig. 9. Positioning errors when using GPS Model 1

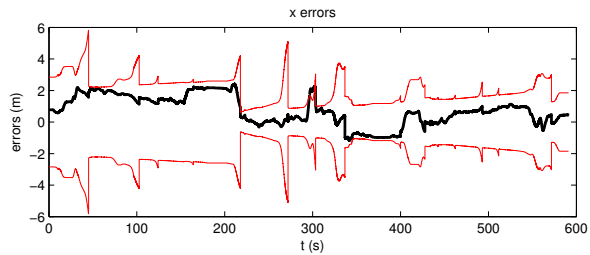

errors

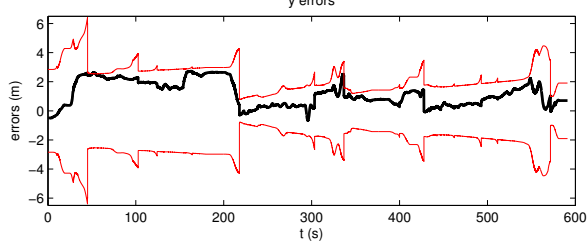

Fig. 10. Positioning errors when using GPS Model 2
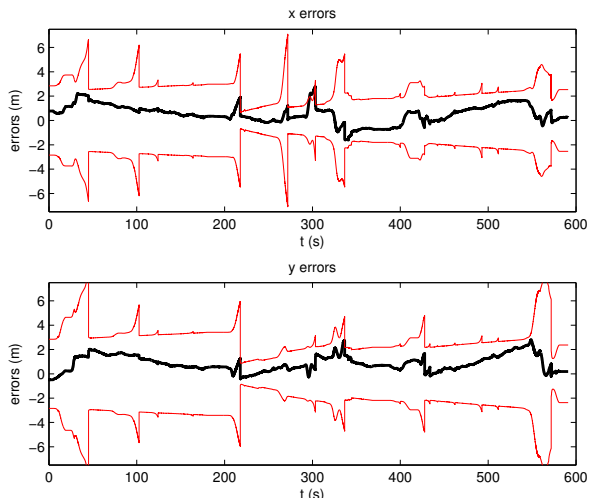

Fig. 11. Positioning errors when using GPS Model 3 $x$ errors
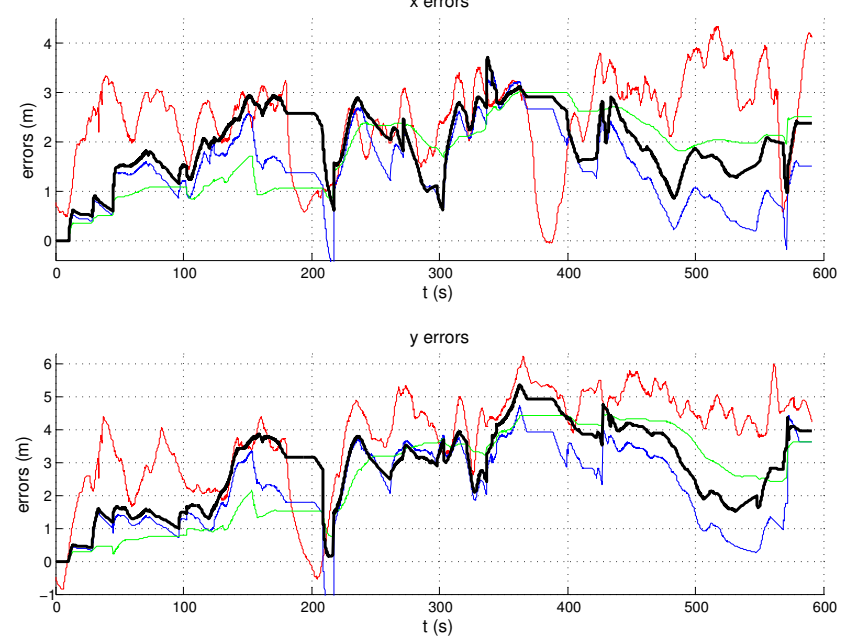

Fig. 12. Estimated GPS errors 